\section{APPAREIL POUR LA MESURE DE LA PUISSANCE DES MOTEURS A GRANDE VITESSE ( $\left.{ }^{\star}\right)$}

L'appareil employé depuis plusieurs années par M. le colonel Renard, pour la mesure de la puissance des moteurs à mouvements rapides (dynamos, moteurs d'automobiles, etc.), est fondé sur les lois de la résistance de l'air. L'inventèr lui a donné le nom de moulinet dynamométrique.

Il consiste simplement en une barre rectangulaire en frêne (fig. I) sur laquelle on boulonne deux plans carrés d'aluminium symétriques par rapport à l'axe $\mathrm{AA}^{\prime}$ autour duquel doit tourner la barre et parallèles à cet axe.

Ces plans peuvent prendre différentes positions indiquées par des chiffres qu'on voit sur la figure. Le chiffre qui définit la position des plans est celui qui tombe en face de la ligne de foi FF' passant par le centre du plan. L'appareil peut être calé directement sur l'arbre du moteur ou sur un arbre auxiliaire placé sur son prolongement et relié au moteur par une bielle de Cardan.

L'expérience montre que, pour une position donnée des plans, le moment moteur est proportionnel au poids spécifique de l'air (que peut donner un abaque) et au carré de la vitesse angulaire.

Si $a$ est le poids spécifique de l'air en kilogrammes par mètre cube; $M$ le moment moteur en kilogrammètres; $N$ le nombre de tours du moulinet par minute et $K_{\mathrm{m}}$ un coefficient appelé coefficient de moment et déterminé par l'expérience, on a :

$$
M=a K_{\mathrm{m}}\left(\frac{N}{1000}\right)^{2}
$$

On en déduit facilement, en désignant par $T$ la puissance en kilogrammètres et par $K_{\mathrm{t}}$ un coefficient dit coefficient de puissance,

$$
T=a K_{\mathrm{t}}\left(\frac{N}{\mathrm{I} 000}\right)^{3}
$$

Les coefficients $K_{\mathrm{t}}$ et $K_{\mathrm{m}}$ sont d'ailleurs reliés par l'équation :

$$
K_{1}=\frac{100 \pi}{3} K_{\mathrm{m}}=104,7^{2} K_{\mathrm{m}}
$$

Les valeurs de $K_{\mathrm{m}}$ ont été déterminées avec précision pour les diverses positions des plans, au moyen d'une balance dynamométrique $\left.{ }^{{ }^{*}}\right)$. On en déduit les valeurs de $K_{t}$.

Il suffit de compter les tours du moulinet pour avoir immédiatement la puissance motrice ou le moment moteur. En employant un tachymètre enregistreur à graduation spéciale, on peut enregistrer directement la puissance puisqu'elle est une fonction de la vitesse seule.

L'apparcil ne s'échauffe pas parce que l'énergie du moteur est absorbée par des masses d'air sans cesse renouvelées. On peut donc prolonger indéfiniment les expériences et étudier avec la plus grande facilité les variations de puissance d'un moteur, car elles se traduisent simplement par des variations de vitesse faciles à enregistrer.

Enfin, des appareils de dimensions restreintes permettent d'essayer des moteurs de grande puissance. Un petit moulinet pesant moins de 2 kilogs peut servir à mesurer une puissance maximum supérieure à 20 chevaux.

Pour rendre la méthode tout à fait pratique, l'inventeur a construit une série de moulinets géométriquement semblables dont la grandeur est définie par un chiffre qu'il

(*) Note de M. le colonel Renard, présentée par M. Maurice Lévy à 'Académie des Sciences, dans la séance du 2 mai 1904.

$\stackrel{(*)}{*}$ Voir plus loin, page 229 , Compte rendu de l'Académie des Sciences. appelle le module. Ce module est l'écartement des trous de la barre en centimètres. La longueur de la barre est égale à vingt-quatre modules; la position des plans est exprimée par leur distance à l'axe en modules, etc.

Chaque module a un diagramme d'emploi (fig. 2) sur lequel on a tracé les paraboles cubiques qui donnent la puissance en chevaux $P$ en fonction de $N$ pour les diffé. rentes positions des plans.

Ce diagramme est limité par un polygone $\mathrm{ABCDE}$, qui indique la limite d'emploi à ne jamais dépasser dans aucun cas. Sur le diagramme de la figure 2 les numéros inscrits sur les courbes sont ceux des divisions de la barre en regard desquelles tombe la ligne médiane tracée sur les plans. Le côté $\mathrm{BC}$ correspond à la vitesse limite à lextrémité de la barre; cette vitesse est fixée à roo mètres par seconde. Le côté $\mathrm{AB}$ est la courbe d'égale tension de la barre. Cette tension maximum est égale à I oo kilogr. par centimètre carré. AE est la parabole cubique correspondant à la position extrême des grands plans (chaque moulinet a deux séries de plans, les grands et les petits) et DC la parabole cubique correspondant à la barre seult.

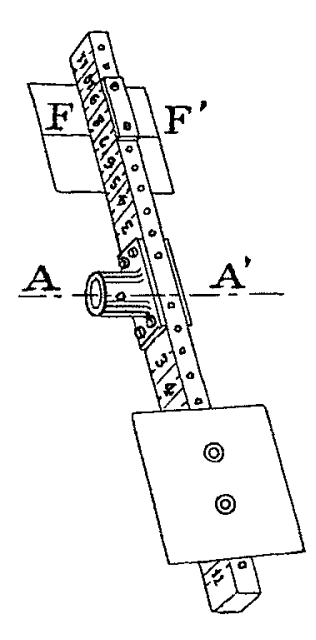

Fig. 1. - Moulinet dynamométrique.

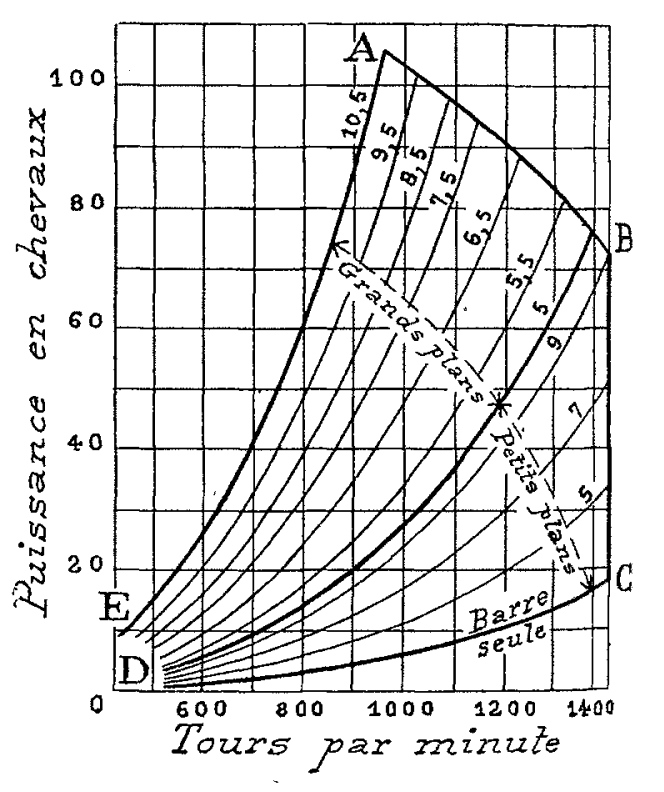

Fig. 2. - Diagramme d'emploi d'un moulinet dynamométrique.
Toute combinaison de $N$ et de $P$ qui donne un point situé à l'intérieur du polygone d'emploi $\mathrm{ABCDE}$ correspond à une expérience réalisable avec le moulinet considéré. Les courbes du diagramme sont tracées en prenant pour la valeur moyenne 1,25 . Une table donne ensuite la correction pour $\mathrm{I} 00$, additive ou soustractive à faire subir à la puissance en fonction de la température et de la pression (température à 1 degré près, pression à 2 millimètres prèss).

La loi suivante permet de déduire tous les coefficients $K_{\text {at }}$ et $K_{\mathrm{t}}$ de leur valeur $\alpha$ et $\beta$ pour le moulinet de module $I$ :

Pour deux moulinets semblables, les cuefficients $K_{\mathrm{m}}$ el $K_{\mathrm{t}}$ sont proportionnels à la cinquieme puissance du module.

Si $p$ est le module, on a donc :

$$
K_{\mathrm{m}}=\alpha \mu^{5}, \quad K_{\mathrm{t}}=\beta \mu^{5} .
$$

Cette nouvelle méthode a la sanction de plusieurs années d'expériences. Elle tend à se généraliser pour les moteurs d'automobiles dont les essais avec les freins ordinaires sont si difficiles. Elle a déjà pu être employée jusqu'ici pour des puissances variant de i à 150 chevaux. Il sera probat blement facile d'aller beaucoup plus loin. 